\title{
Universal Testing of all Pregnant Women for COVID- 19: A Way Forward for Comprehensive Antenatal Care
}

\author{
Hema Divakar *, Poorni Narayanan, Rita Singh, Divakar Gubbi Jyothi Girish \\ Divakars Speciality Hospital, Bengaluru, 560078, India \\ Correspondence should be addressed to Hema Divakar; artistbengaluru@ gmail.com
}

Asian Research and Training Institute for Skill Transfer (ARTIST), Centre of Excellence for Women's Health Care,

Received 08 August 2020;

Accepted 30 August 2020;

Published 01 September 2020

\begin{abstract}
The COVID-19 global pandemic has placed undue stress on health systems all around the world. However, little is known about the impact of exposure to the virus on growing foetuses or the course of COVID-19 in pregnant women, who are often asymptomatic. To develop effective policies and recommendations, robust data for both asymptomatic and symptomatic pregnant women is crucial. This can only be acquired through universal testing of all pregnant women for COVID-19. The acquired data will not only enhance our ability to answer questions around negative obstetric outcomes, such as miscarriages and preterm labours, but also lead to enhanced contact tracing, which will slow the spread of COVID-19.
\end{abstract}

Keywords: Covid 19, pregnancy, testing:ing:

\section{Introduction}

Since its outbreak in Wuhan, China, new coronavirus 2019 (COVID-19), a disease caused by the severe acute respiratory syndrome coronavirus 2 (SARS-CoV-2), has been declared a global pandemic ${ }^{[1]}$, with over 18 million confirmed cases to date [2].

Health systems around the world, especially those in lowand middle-income countries (LMIC), have been overwhelmed by the large volumes of patients, including pregnant women, presenting with COVID-19 symptoms ${ }^{[3,4]}$. Fear, stigma, misinformation, and limitations on movement that disrupt the delivery of health care have further compounded challenges faced in meeting increasing demand for the care of pregnant women with COVID-19, including those who are asymptomatic.

Until recently, pregnant women in India were tested for COVID-19 only if they had symptoms, known exposures, or history of recent travel to COVID-19 hot spots ${ }^{[5]}$. However, recent advisory from the Indian Council of Medical Research (ICMR) mandates testing of all pregnant women in labour or close to term, even if they have no symptoms. We believe that a strategy including universal testing of all pregnant women presenting at obstetric departments, irrespective of gestational age, symptoms, or known exposure, in addition to those who present for triage evaluation of symptomatic complaints, has obvious benefits that should inform best practices to protect patients, their families, and obstetrical providers:

a) Studies ${ }^{[6]}$ have revealed that pregnant women diagnosed with COVID-19 in the third trimester develop complications. Furthermore, infants born to these women have tested negative for Sars-COV-2 despite developing non-severe neonatal complications; however, it is not clear whether the incidence of neonatal complications is similar to that in infants born to women who do not test positive for COVID-19 ${ }^{[6]}$. A more robust dataset would allow us to study the trends of adverse events throughout pregnancy and analyse the impact of COVID-19 in pregnant women. To enhance our ability to answer questions around miscarriages, materno-fetal transmission, birth defects, bleeding in pregnancy, and preterm labours, more data on the course of COVID-19 in pregnant women is needed.

b) Enhanced testing will provide the necessary alerts for isolation and further monitoring of those who had come in contact with asymptomatic pregnant women. Furthermore, larger cluster of contacts can be traced and tested to prevent spread. Decisions should be aligned with relevant national and subnational policies, and should be re-evaluated at regular intervals.

Here, we report an asymptomatic case of COVID-19 infection in a pregnant woman presenting to a large private hospital in India.

\section{Case Report}

Mrs. X, a old primigravida with a live singleton foetus with an appropriate growth profile, had her routine antenatal check at 32 weeks' gestation. She was found to have no co-morbidities and was therefore considered at low risk for pregnancy complications. 
One week after this routine check, a family friend visited her at her home. The very next day, the friend was reported to have complained of a fever and breathlessness. She was subjected to a COVID-19 test, which was positive. Even though Mrs X was asymptomatic, as a traced contact, she was tested by the authorities for COVID-19. An RTPCR throat swab test revealed that she was positive. The health authorities considered her to be high risk because she was pregnant and advised admission at a COVID-19 care centre.

After admission, she underwent daily monitoring for development of symptoms associated with COVID-19 infection, including a fever, upper respiratory infection symptoms, anosmia, and hypogeusia. Furthermore, monitoring of temperature rise and oxygen saturation, foetal heartbeat and foetal movements, and uterine contractions were also performed. Two days later, she developed anosmia in the absence of congestion and other nasal symptoms, although she had no history of hyposmia or anosmia, and hypogeusia. The course in the hospital was uneventful. On day 14, after a COVID-19 test returned a negative result, she was discharged.

Three of her family members (her contacts), who lived in her own home, all tested positive for COVID-19, even though they were asymptomatic. Home isolation was recommended as well as digital monitoring. A re-test for COVID-19 after 14 days returned negative results for all of her family members.

Mrs. X presented with rupture of the membranes at 38 weeks. A C section was performed under regional anaesthesia for obstetric indication. She delivered a live female baby weighing 3.2 $\mathrm{kg}$ with good Apgar scores. The amniotic fluid was clear, and the gross appearance of the placenta showed no areas of haemorrhage, infarct, or fibrosis. The baby was roomed in with the mother, who breastfed the baby successfully with adherence to hand hygiene and masking. Covid test not done for the newborn as it is not mandated as per ICMR guidelines Routine care and routine immunization was offered.

Though anxious and apprehensive at admission, Mrs. X did not show any signs of overt anxiety or depression, and did not need a formal counselling session nor a visit by the mental health expert. Her post-operative course was uneventful, and outcomes were good both for the mother and baby. Prior to discharge, the mother and the infant did not exhibit any new symptoms. At the one-week follow up, the original symptoms of anosmia had not recurred; furthermore, there were no noteworthy complaints.

\section{Discussion}

The physiological and immunological changes that occur during pregnancy increase the risk of poor obstetric outcomes in pregnant women in the event of respiratory infection ${ }^{[7]}$. In the previous coronavirus outbreaks of severe acute respiratory syndrome (SARS) and Middle East respiratory syndrome (MERS), for example, pregnant women were more likely to develop severe complications that led to increased mortality, morbidity, and hospitalization ${ }^{[7,8]}$. However, the limited available data on the course of COVID-19 in pregnant women have shown pregnant women with COVID-19 are often asymptomatic ${ }^{[9]}$. Furthermore, according to a report on 43 cases treated at Columbia University Irving Medical Center and the Allen Hospital, New York, USA ${ }^{[10]}$, nearly nine out of 10 pregnant women will develop only a mild disease, based on the criteria developed by $\mathrm{Wu}$ and McGoogan ${ }^{[11]}$. Studies seem to also suggest that COVID-19 infection in pregnant women could, nevertheless, potentially increase hospitalizations. A comparison of the outcomes in pregnant patients with those in
83,205 nonpregnant women with COVID-19 revealed that there was a substantially greater proportion of hospital admissions among pregnant patients (31.5\%) compared with nonpregnant patients (5.8\%) with COVID-19, although the authors could not distinguish between hospitalizations due to COVID-19 and those arising from pregnancy, including delivery ${ }^{[12]}$.

The role that asymptomatic infections play in the spread of COVID-19 is also a subject of increasing scrutiny. It has been estimated that up to $40-45 \%$ of all people with COVID-19 are asymptomatic yet potentially infectious ${ }^{[13]}$. Among residents at a skilled nursing facility in Washington State, Seattle, USA, more than half who were asymptomatic at testing (27/48) eventually developed symptoms ${ }^{[14]}$. Moreover, at the time of testing, their viral loads were as high as those of residents who were symptomatic, indicating their potential for viral transmission was high ${ }^{[14]}$. In pregnant women, asymptomatic rates as low as $1 \%$ in hospitals in Boston ${ }^{[15]}$ to as high as $14 \%$ in hospitals in New York ${ }^{\text {[9] }}$ have been reported, with the wide disparity being attributed to differences in population density, COVID-19 community mitigation and containment methods, and the overall prevalence of COVID-19 within the community. These findings indicate that identification, isolation, and monitoring of asymptomatic cases could not only reduce the spread of COVID-19, but inform and improve its clinical management, especially in pregnant women, leading to better outcomes. The American College for Obstetricians and Gynaecologists is therefore reviewing all its COVID-19-related clinical and patient materials, and declared that it will make any necessary revisions to recommendations ${ }^{[16]}$. In the meantime, owing to the many unknowns regarding SARS-CoV-2 infection in pregnant women, clinicians in India have been advised to alert patients to the potential increased risk for severe COVID19 symptoms during pregnancy. They have also been advised to stress to pregnant women and their families the need for precautions to prevent infection, paying particular attention to measures to protect those with greater occupational exposure to the virus.

These recommendations notwithstanding, in order to provide the right guidance and advice to obstetric patients and pursue the most appropriate interventions, we need robust data, which can only be acquired through increased testing. It is imperative that we expand testing capacity to include all pregnant women even if they are asymptomatic. Although studies performed on women at 36 weeks' gestation and beyond have revealed the effects of COVID-19 on late-stage pregnancy ${ }^{[17,18]}$, we do not know the potential impact of exposure to the virus in first and second trimesters. Furthermore, although it has been established that proinflammatory cytokines, such as interleukin 6 (IL-6), that are elevated in COVID-19 patients are also elevated in pregnancy, the effect of elevated IL- 6 levels on the course of COVID-19 in pregnant women is unknown. In addition, the placenta possess angiotensin-converting enzyme 2 (ACE2) receptors through which COVID-19 has been shown to enter host cells ${ }^{[19]}$. While available data suggest that vertical transmission of COVID-19 is uncommon ${ }^{[17]}$, it is not known whether the presence of ACE2 receptors on the placenta negatively influences foetal and placenta health in symptomatic and asymptomatic pregnant women. For example, is risk of embryonic or foetal demise increased due to the presence of SARS-CoV-2 on the placenta?

A COVID-19 antigen test can fill an important gap in the testing landscape by providing fast diagnoses in the clinic, but it is not perfect due to its high false negative rate ${ }^{[20]}$. However, when combined with more accurate PCR tests and blood tests that look for antibodies, antigen testing could play a large role in helping 
public health officials better understand and limit the spread of COVID-19 in pregnant women. Although obstetric practitioners recognize that it would be helpful to obtain a COVID-19 test for all their patients, especially those in the second or third trimester, testing policies limiting testing to patients with respiratory symptoms make this difficult. In the future, as antibody tests become more available, it may be useful to re-test pregnant women who experienced negative obstetric outcomes for COVID-19 antibodies to ascertain whether they were exposed to the virus and if SARS-CoV-2 infection was a contributing factor. Furthermore, a prospective multicenter follow up of all COVID-19 positive cases as well as matched controls (age, parity, socioeconomic status, and obstetric profile) would be equally informative.

\section{Conclusions}

The lack of robust data on the course of COVID-19 in pregnant women and its impact on foetal health has caused anxiety among pregnant women and health care providers, in addition to negatively impacting the delivery of quality care. Furthermore, it is currently not known, despite the low levels of mortality due to COVID-19 in this population, whether COVID-9 contributes to negative obstetric outcomes, such as miscarriages or premature delivery, and whether these outcomes vary between symptomatic and asymptomatic women. Universal screening of pregnant women would identify asymptomatic pregnant women, limiting the spread of SARS-CoV-2 infection to their caregivers and healthcare providers, and later to neonates in the postpartum period. Furthermore, it will inform clinical management of COVID-19 in pregnant women, informing policy and recommendations. Coupled with contact tracing, rapid isolation, and supportive quarantine, it could slow the spread of COVID-19, especially now as lockdowns are being eased throughout India. Successful implementation of these strategic shifts will require the active engagement of communities and public and private stakeholders, specific measures to ensure access for socially vulnerable populations, transparency and frequent communication with the public, and a high degree of cooperation from individuals.

\section{Ethics approval and consent to participate}

"Not applicable".

\section{Conflicts of Interest}

The authors declare that there are no conflict of interest regarding the publication of this paper.

\section{Funding Statement}

"Not Applicable"

\section{Authors}

H.D., P.N., R.S., G. D., and G J.contributed to the writing of the manuscript

\section{References}

[1] WHO. (2020) WHO announces COVID-19 outbreak a pandemic. https://www.euro.who.int/en/healthtopics/health-emergencies/coronavirus-COVID-
19/news/news/2020/3/who-announces-COVID-19outbreak-a-pandemic

[2] Dong E, Du H, Gardner L. An interactive web-based dashboard to track COVID-19 in real time. Lancet Infectious Diseases. 20(5):533-534.

[3] AFP. 2020. It's exploding: Overwhelmed Mumbai hospitals near collapse. https://www.freemalaysiatoday.com/category/world/2020 /05/16/its-exploding-overwhelmed-mumbai-hospitalsnear-collapse/

[4] Dutt, A. 2020. With hospitals overwhelmed, pregnant women left with no care or place to give birth. Hindustan Times. https://www.hindustantimes.com/delhinews/with-hospitals-overwhelmed-pregnant-women-leftwith-no-care-or-place-to-give-birth/storyiqOmJLgWn4TyXwx719O8vM.html

[5] Indian Council of Medical Research (ICMR). Guidance for Management of Pregnant Women in COVID-19 Pandemic.

(2020).https://www.icmr.gov.in/pdf/covid/techdoc/Guida nce_for_Management_of_Pregnant_Women_in_COVID 19_Pandemic_12042020.pdf

[6] Schwartz DA. An analysis of 38 pregnant women with COVID-19, their newborn infants, and maternal-fetal transmission of SARS-CoV-2: maternal coronavirus infections and pregnancy outcomes. Archives of Pathology and Laboratory Medicine. 2020 Jul;144(7):799-805.

[7] Jamieson DJ, Honein MA, Rasmussen SA. H1N1 2009 influenza virus infection during pregnancy in the USA. Lancet. 2009;374:451-458

[8] Wong SF, Chow KM, Leung TN. Pregnancy and perinatal outcomes of women with severe acute respiratory syndrome. American Journal of Obstetrics and Gynaecology. 2004;191:292-297.

[9] Sutton D, Fuchs K, D'alton M, Goffman D. Universal screening for SARS-CoV-2 in women admitted for delivery. New England Journal of Medicine. 2020 May 28;382(22):2163-4.

[10] Breslin N, Baptiste C, Gyamfi-Bannerman C, et al. COVID-19 infection among asymptomatic and symptomatic pregnant women: Two weeks of confirmed presentations to an affiliated pair of New York City hospitals. American Journal of Obstetrics and Gynaecology MFM. 2020 Apr; 9:100118.

[11] $\mathrm{Wu}, \mathrm{Z}$ and McGoogan, JM. Characteristics of and important lessons from the coronavirus disease 2019 (COVID-19) outbreak in China: summary of a report of 72314 cases from the Chinese Centre for Disease Control and Prevention. JAMA 2020;323: 1239-1242.

[12] Ellington S, Strid P, Tong VT, et al. Characteristics of Women of Reproductive Age with LaboratoryConfirmed SARS-CoV-2 Infection by Pregnancy Status United States, January 22-June 7, 2020. Morbidity and Mortality Weekly Report. 2020; 69:769-775.

[13] Oran DP and Topol EJ. Prevalence of Asymptomatic SARS-CoV-2 Infection: A Narrative Review. Annals of Internal Medicine. 2020 Jun 3.

[14] Arons MM, Hatfield KM, Reddy SC, et al. Presymptomatic SARS-CoV-2 infections and transmission in a skilled nursing facility. New England Journal of Medicine 2020; 382:2081-2090. 
[15] Goldfarb IT, Diouf K, Barth WH, et al. Universal SARSCoV-2 testing on admission to the labor and delivery unit: low prevalence among asymptomatic obstetric patients. Infectious Control Hospital Epidemiology. Published online June 15, 2020.

[16] ACOG. 2020. General Information Regarding Pregnant Individuals and COVID-19.

https://www.acog.org/clinical/clinical-guidance/practiceadvisory/articles/2020/03/novel-coronavirus-2019

[17] Chen H, Guo JMS, Chen W, et al. Clinical characteristics and intrauterine vertical trans- mission potential of COVID-19 infection in nine pregnant women: a retrospective review of medical records. Lancet. 2020;395(10226): 809-15.

[18] Yu N, Li W, Kang Q, Xiong Z, Wang S, Lin X, Liu Y, Xiao J, Liu H, Deng D, Chen S. Clinical features and obstetric and neonatal outcomes of pregnant patients with COVID-19 in Wuhan, China: a retrospective, singlecentre, descriptive study. The Lancet Infectious Diseases. 2020 Mar 24.

[19] Dashraath P, Jeslyn WJ, Karen LM, Min LL, Sarah L, Biswas A, Choolani MA, Mattar C, Lin SL. Coronavirus disease 2019 (COVID-19) pandemic and pregnancy. American Journal of Obstetrics and Gynaecology. 2020 Mar 23.

[20] Ai T, Yang Z, Hou H, Zhan C, Chen C, Lv W, Tao Q, Sun Z, Xia L. Correlation of chest CT and RT-PCR testing in coronavirus disease 2019 (COVID-19) in China: a report of 1014 cases. Radiology. 2020 Feb 26:200642. 